\title{
Chromospheric activity on the RS Canum Venaticorum binary SZ Piscium
}

\author{
L.-Y. Zhang ${ }^{1,2}$ and S.-H. Gu ${ }^{1}$ \\ 1 National Astronomical Observatories/Yunnan Observatory, Joint laboratory for Optical Astronomy, Chinese Academy of Sciences, \\ Kunming 650011, PR China \\ e-mail: liy_zhang@hotmail.com \\ 2 Graduate School of Chinese Academy of Sciences, Beijing 100039, PR China
}

Received 29 February 2008 / Accepted 10 June 2008

ABSTRACT

\begin{abstract}
Aims. We present the new high-resolution echelle spectra of SZ Psc, obtained in Nov. 2004 and Sep.-Dec. 2006, and study its chromospheric activity.

Methods. By means of the spectral subtraction technique, we analyze our spectroscopic observations including several optical chromospheric activity indicators (the $\mathrm{He}$ I $\mathrm{D}_{3}, \mathrm{Na}$ I $\mathrm{D}_{1}, \mathrm{D}_{2}, \mathrm{H}_{\alpha}$, and $\mathrm{Ca}$ II infrared triplet lines).

Results. All indicators show that the chromospheric activity of the system is associated with the cooler component. We find that the values of $E W_{8542} / E W_{8498}$ are in the range 1-3, which indicates optically thick emission in plage-like regions. The 2006 data suggest the presence of active longitude phenomena. For the Ca II 8542 and 8662 and the $\mathrm{H}_{\alpha}$ lines, it seems that the excess emission is stronger near the two quadratures of system. This may be anti-correlated with the behavior of the $\mathrm{Na} I \mathrm{D}_{1}$ line. The absorption features are detected in the subtracted $\mathrm{H}_{\alpha}$ lines, which could be explained by prominence-like extended material seen on the stellar disk or by mass transfer from the cooler component to the hotter one.
\end{abstract}

Key words. stars: binaries: eclipsing - stars: binaries: spectroscopic - stars: chromospheres - stars: activity - stars: late-type stars: individual

\section{Introduction}

SZ Psc (HD 219113, $P_{\text {orb }}=3.97$ ) is a partially eclipsing and double-lined spectroscopic binary consisting of F8V-IV hotter and K1IV cooler components (Jakate et al. 1976). Hall (1976) defined it as a typical member of RS CVn binaries, which are characterized by strong magnetic activity that presents as photospheric spots, chromospheric emission, transition region emission, coronal X-ray radiation, and flare events.

The first variability of the lightcurve of SZ Psc was found by Jensch (1934). Since then, the light-curve variations have been analyzed by many authors (Jakate et al. 1976; Eaton 1977; Catalano et al. 1978; Eaton et al. 1982; Tunca 1984; Doyle et al. 1994a; Antonopoulou et al. 1995; Lanza et al. 2001; Kang et al. 2003; Eaton \& Henry 2007). For the distortion wave on the lightcurves of SZ Psc, this has been explained by the starspot model (Eaton \& Hall 1979; Lanza et al. 2001; Kang et al. 2003; Eaton \& Henry 2007).

The spectroscopic observations have been carried out by many groups (Jakate et al. 1976; Bopp \& Talcott 1978; Weiler 1978; Bopp 1981; Ramsey \& Nations 1981; Huenemoerder \& Ramsey 1984; Fernández-Figueroa et al. 1986; Popper 1988; Fernández-Figueroa et al. 1994; Frasca \& Catalano 1994; Montes et al. 1995; Eaton \& Henry 2007). Jakate et al. (1976) and Popper (1988) derived the spectroscopic orbital elements on the basis of the radial velocity curves, and they also found strong chromospheric emission in the $\mathrm{Ca}$ II $\mathrm{H} \& \mathrm{~K}$ lines from the cooler component. A flare-like phenomenon was detected in the $\mathrm{Ca}$ II $\mathrm{H}$ \& K lines by Fernández-Figueroa et al. (1986). Recently, Eaton $\&$ Henry (2007) have revised the orbital elements of the system, but so far there has been no spectroscopic study of the Ca II IRT lines for SZ Psc.

SZ Psc is very active, because of its extremely variable $\mathrm{H}_{\alpha}$ profiles. In 1978, Weiler (1978) found that SZ Psc only exhibited significant variation in its $\mathrm{H}_{\alpha}$ emission lines near orbital phase 0.8. Moreover, Bopp \& Talcott (1978) confirmed this result and found $\mathrm{H}_{\alpha}$ emission only in the phase range 0.6-0.8. However, Ramsey \& Nations (1981) found $\mathrm{H}_{\alpha}$ emission is in the phase range $0-0.4$ and interpreted the $\mathrm{H}_{\alpha}$ behavior as a flare event that locally injects material into a transient disk or shell structure around the K1 IV star. Afterward, Bopp (1981) discussed an unusual $\mathrm{H}_{\alpha}$ profile, particularly its large width and double-peaked appearance. Beyond that, Huenemoerder \& Ramsey (1984) found that SZ Psc was highly active with the emission from a cooler component and that it had undergone a large $\mathrm{H}_{\alpha}$ outburst during and after which the profiles were suggestive of a circumstellar origin. The extremely variable $\mathrm{H}_{\alpha}$ profiles suggest there are two active longitudes. Recently, Eaton \& Henry (2007) have found that $\mathrm{H}_{\alpha}$ emission seems independent of the orbital phase on average, but it can increase markedly within a few orbital cycles.

Up to now, the spectral subtraction technique has been used widely for chromospherically active binary systems in several lines: $\mathrm{Ca}$ II $\mathrm{H} \& \mathrm{~K}, \mathrm{Mg} \mathrm{I} \mathrm{b}, \mathrm{He} \mathrm{I} \mathrm{D}_{3}$, Na I $\mathrm{D}_{1}, \mathrm{D}_{2}, \mathrm{H}_{\alpha}, \mathrm{H}_{\beta}$, Ca II IRT (Gunn \& Doyle 1997; Gunn et al. 1997; Lázaro \& Arévalo 1997; Montes et al. 1997; Arévalo \& Lázaro 1999; Frasca et al. 2000; Montes et al. 2000; Shan et al. 2006; Gálvez et al. 2007, etc.). The technique was described in detail for obtaining the chromospheric contribution by Barden (1984) and Montes et al. (1995). Also, it was discussed and applied in the 

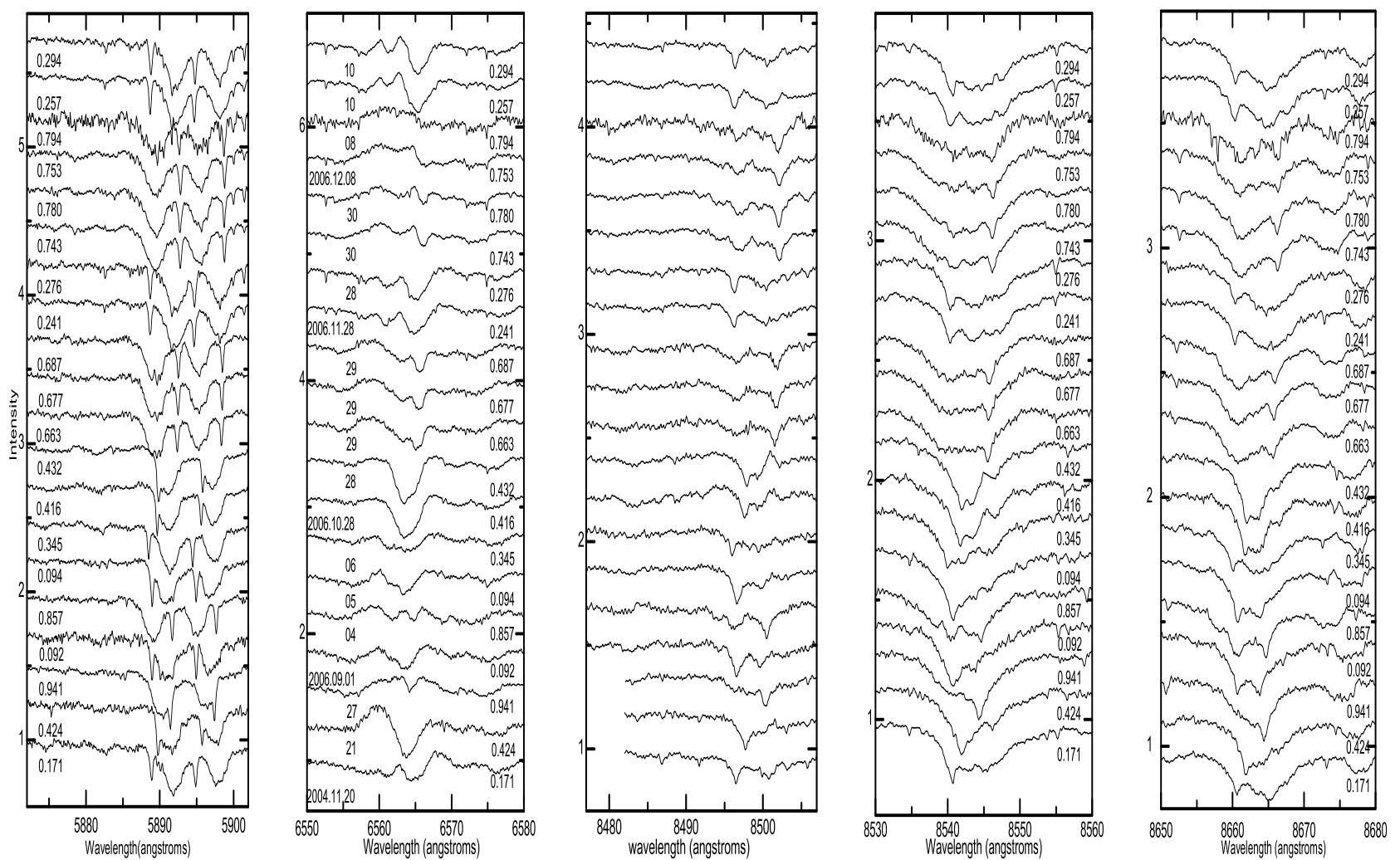

Fig. 1. The $\mathrm{He} \mathrm{I}_{3}$, Na I $\mathrm{D}_{1}, \mathrm{D}_{2}, \mathrm{H}_{\alpha}$, and Ca II IRT line profiles of SZ Psc.

search for prominence-like events (Gunn \& Doyle 1997; Gunn et al. 1997).

In this paper, new spectroscopic observations of SZ Psc are analyzed using the spectral subtraction technique. Some new results are derived for the chromospherically active activity of SZ Psc.

\section{Observation}

The new spectroscopic observations of SZ Psc were carried out with the $2.16 \mathrm{~m}$ telescope at the Xinglong station of the National Astronomical Observatories, China, in five observing runs: Nov. 20-27, 2004, Sep. 1-6, 2006, Oct. 28-29, 2006, Nov. 28-30, 2006, and Dec. 8-10, 2006. The Coudé echelle spectrograph with a spectral resolution about 37000 and a $1024 \times 1024$ pixel Tektronix CCD detector were used during our observations. The reciprocal dispersions are $0.079 \AA$ A/pixel for the $\mathrm{He} \mathrm{ID}_{3}, \mathrm{Na} \mathrm{ID}_{1}, \mathrm{D}_{2}$ spectral region, $0.089 \AA /$ pixel for the $\mathrm{H}_{\alpha}$ spectral region, $0.113 \AA$ /pixel for the Ca II 8498 spectral region, $0.115 \AA$ /pixel for the Ca II 8542 spectral region, and $0.117 \AA$ /pixel for the Ca II 8662 spectral region. Correspondingly, the spectral resolution determined as the FWHM of the arc comparison lines is $0.150,0.160,0.207,0.218$, and $0.221 \AA$, respectively.

The spectra were reduced using the IRAF package. The standard procedures were used, which include image trimming, bias subtraction, flat-field division, background subtraction, cosmic ray removal, and spectrum extraction. The wavelength calibration was obtained by taking the spectra of a Th-Ar lamp. Finally, the spectra were normalized by a polynomial fit to the observed continuum. The normalized spectral profiles are displayed in Fig. 1. During our observations, the signal-to-noise ratio $(S / N)$
Table 1. The observational log of SZ Psc.

\begin{tabular}{ccccccccc}
\hline \hline Date & \multicolumn{2}{c}{ HJD } & \multicolumn{3}{c}{ Phase Exp. time } & \multicolumn{5}{c}{$S / N$} \\
\cline { 3 - 9 } & $2450000+$ & & $(\mathrm{s})$ & $\mathrm{Na}_{\mathrm{I}} \mathrm{H}_{\alpha}$ & $\lambda 8498$ & $\lambda 8542$ & $\lambda 8662$ \\
\hline 2004.11 .20 & 3330.1056 & 0.171 & 2400 & 122 & 169 & 146 & 172 & 158 \\
2004.11 .21 & 3331.1074 & 0.424 & 2400 & 72 & 102 & 104 & 121 & 111 \\
2004.11 .27 & 3337.1254 & 0.941 & 2400 & 92 & 122 & 105 & 119 & 109 \\
2006.09 .01 & 3980.1606 & 0.092 & 3600 & 60 & 89 & 114 & 125 & 119 \\
2006.09 .04 & 3983.1945 & 0.857 & 4200 & 107 & 137 & 136 & 146 & 137 \\
2006.09 .05 & 3984.1343 & 0.094 & 3600 & 121 & 161 & 159 & 173 & 162 \\
2006.09 .06 & 3985.1275 & 0.345 & 3600 & 148 & 197 & 191 & 210 & 195 \\
2006.10 .28 & 4036.9631 & 0.416 & 3600 & 159 & 208 & 190 & 211 & 198 \\
2006.10 .28 & 4037.0292 & 0.432 & 3600 & 174 & 231 & 216 & 241 & 225 \\
2006.10 .29 & 4037.9423 & 0.663 & 3600 & 105 & 137 & 130 & 141 & 131 \\
2006.10 .29 & 4037.9973 & 0.677 & 3600 & 101 & 134 & 130 & 141 & 131 \\
2006.10 .29 & 4038.0403 & 0.687 & 3600 & 105 & 139 & 138 & 149 & 141 \\
2006.11 .28 & 4067.9936 & 0.241 & 3000 & 105 & 136 & 135 & 150 & 140 \\
2006.11 .28 & 4068.1349 & 0.276 & 2400 & 76 & 107 & 110 & 122 & 114 \\
2006.11 .30 & 4069.9877 & 0.743 & 3000 & 110 & 142 & 135 & 148 & 138 \\
2006.11 .30 & 4070.1315 & 0.780 & 3000 & 92 & 136 & 133 & 152 & 140 \\
2006.12 .08 & 4077.9560 & 0.753 & 3600 & 82 & 108 & 106 & 118 & 110 \\
2006.12 .08 & 4078.1179 & 0.794 & 3600 & 37 & 52 & 55 & 61 & 58 \\
2006.12 .10 & 4079.9561 & 0.257 & 3600 & 123 & 162 & 157 & 173 & 161 \\
2006.12 .10 & 4080.1039 & 0.294 & 3600 & 92 & 136 & 136 & 152 & 141 \\
\hline
\end{tabular}

varied in quality, and in some instances it suffered from poor seeing and intermittent clouds. For most of the observations, the $S / N$ is more than 100. The observing log is listed in Table 1 , which includes the observing date, the Heliocentric Julian Date of observation (HJD), orbital phase, exposure time, and the $S / N$. The orbital phases were calculated using the revised ephemeris derived by Eaton \& Henry (2007).

On some nights of our observing seasons when the telluric lines were heavy, two rapidly rotating early-type stars, 

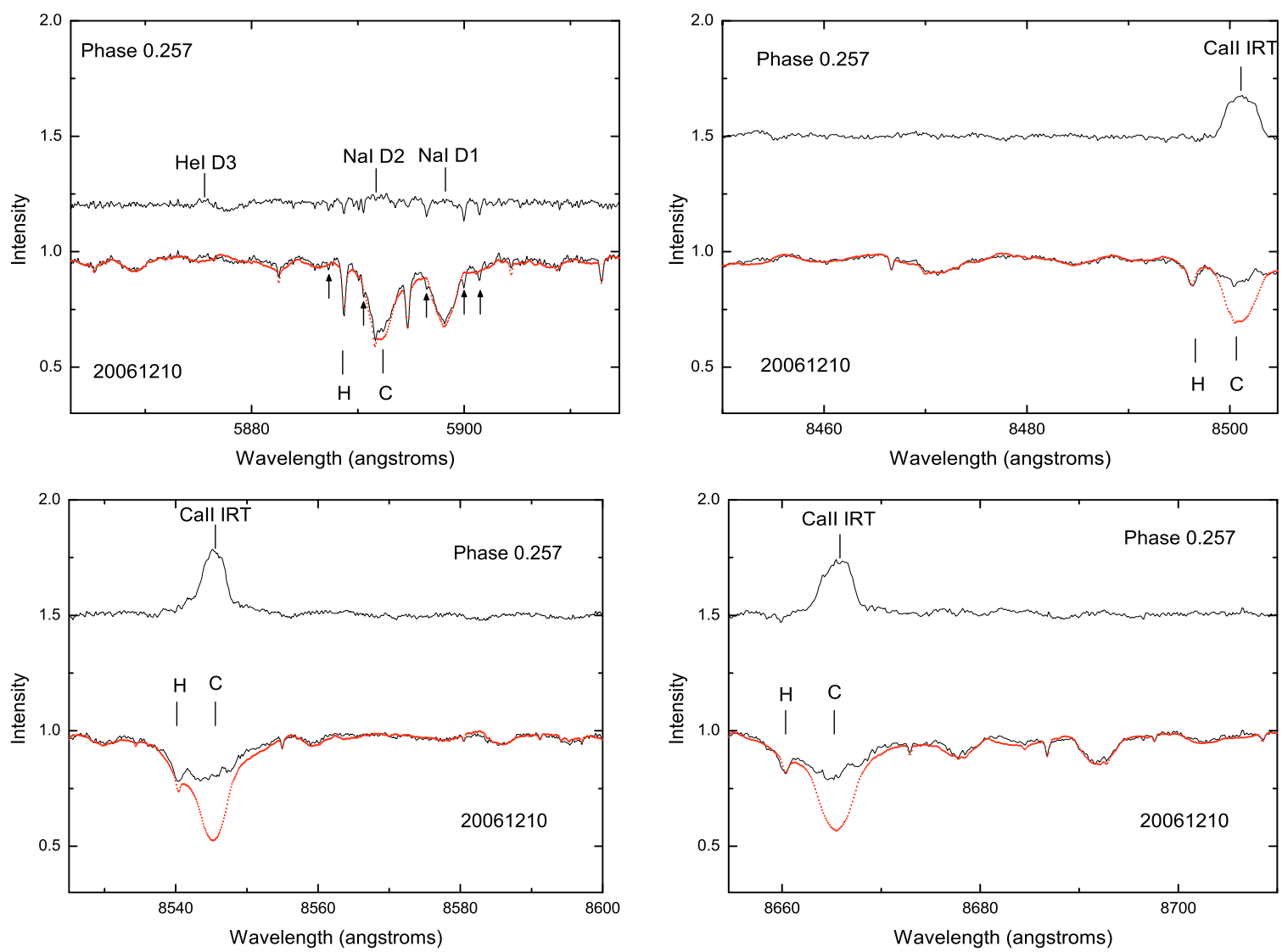

Fig. 2. Samples of the observed, synthesized, and subtracted spectra for the He I $\mathrm{D}_{3}, \mathrm{Na} \mathrm{I}_{1}, \mathrm{D}_{2}$, and Ca II IRT lines. The dotted lines represent the synthesized spectra and the upper spectra are the subtracted spectra. Vertical arrows mark the telluric lines that appeared in the spectral region.

HR $7894\left(\mathrm{~B} 5 \mathrm{IV}, v \sin i=330 \mathrm{~km} \mathrm{~s}^{-1}\right)$ and HR $989(\mathrm{~B} 5 \mathrm{~V}, v \sin i=$ $298 \mathrm{~km} \mathrm{~s}^{-1}$ ), were observed. Their observed spectra were fitted using the high-order spline 3 function to derive the telluric line templates. For the spectra of HR 989, interstellar Na I lines were present and removed. The telluric lines in the spectra of SZ Psc were removed by means of these templates obtained on the same nights. The examples of this procedure in different spectral regions were given by Gu et al. (2002).

\section{Spectral analysis}

The normalized spectra of SZ Psc are analyzed using the spectral subtraction technique. In this method, the synthesized spectra is constructed from artificially rotationally broadened, radialvelocity shifted, and weighted spectra of two inactive stars with the same spectral type and luminosity class as the two components of the active system. For our situation, the synthesized spectrum is constructed by means of the program STARMOD (Barden 1985). We observed several stars with spectral types and luminosity classes similar to those of the individual components of SZ Psc. By comparison, we find that two inactive stars HR 7948 (K1IV) and HR 6669 (F8V) are much better templates for SZ Psc. Thus, we chose them as template stars to construct synthesized spectra for all spectral data of SZ Psc. For the hotter component, Strassmeier et al. (1993) gave F8 IV for the spectral type. However, we find that HR 6669 is a better template for the hotter component. Moreover, HR 6669 does have the advantage of being an extremely inactive star (Wright et al. 2004), and Gray et al. (2001) gave F8 V for its spectral type, so the spectral type of the hotter component should be F8V.
In the course of the analysis, the $v \sin i$ values $\left(0 / 78 \mathrm{~km} \mathrm{~s}^{-1}\right)$ of the components of SZ Psc were used, which are determined from the spectra spanning the wavelength ranges 6389-6477 and 6615-6706 $\AA$ with many photospheric lines. These values are close to $80 \mathrm{~km} \mathrm{~s}^{-1}$ for the cooler component and less than $5 \mathrm{~km} \mathrm{~s}^{-1}$ for the hotter component, as published by Eaton \& Henry (2007). The intensity weight for each of the two components is obtained from high-quality spectra around phases 0.25 and 0.75 where the two components are separated well. Consequently, the adopted intensity weight ratios are $0.25 / 0.75$ for $\mathrm{He} \mathrm{I}_{3}$ and $\mathrm{Na} \mathrm{I}_{1}, \mathrm{D}_{2}$ spectral region, 0.20/0.80 for $\mathrm{H}_{\alpha}$ spectral region, 0.18/0.82 for the Ca II 8498 spectral region, $0.175 / 0.825$ for the Ca II 8542 spectral region, and $0.17 / 0.83$ for the Ca II 8662 spectral region.

Samples of the synthesized and subtracted spectra (the observed spectra minus the synthesized one) obtained in the He I $\mathrm{D}_{3}, \mathrm{Na} \mathrm{I} \mathrm{D}_{1}, \mathrm{D}_{2}$, and $\mathrm{Ca}$ II IRT line regions are displayed in Fig. 2. Since the $\mathrm{H}_{\alpha}$ features are bizarre, some samples are displayed in Fig. 3. For each sample, we plot the observed (lower solid line), the synthesized (dashed line), and the subtracted spectra (upper solid line), which is offset for better display. In these figures, " $\mathrm{H}$ " and " $\mathrm{C}$ " represent the hotter and cooler components, respectively.

\section{Chromospheric activity indicators}

The new spectroscopic observations allow us to simultaneously analyze the behavior of the different chromospheric activity indicators formed at different atmospheric heights. 

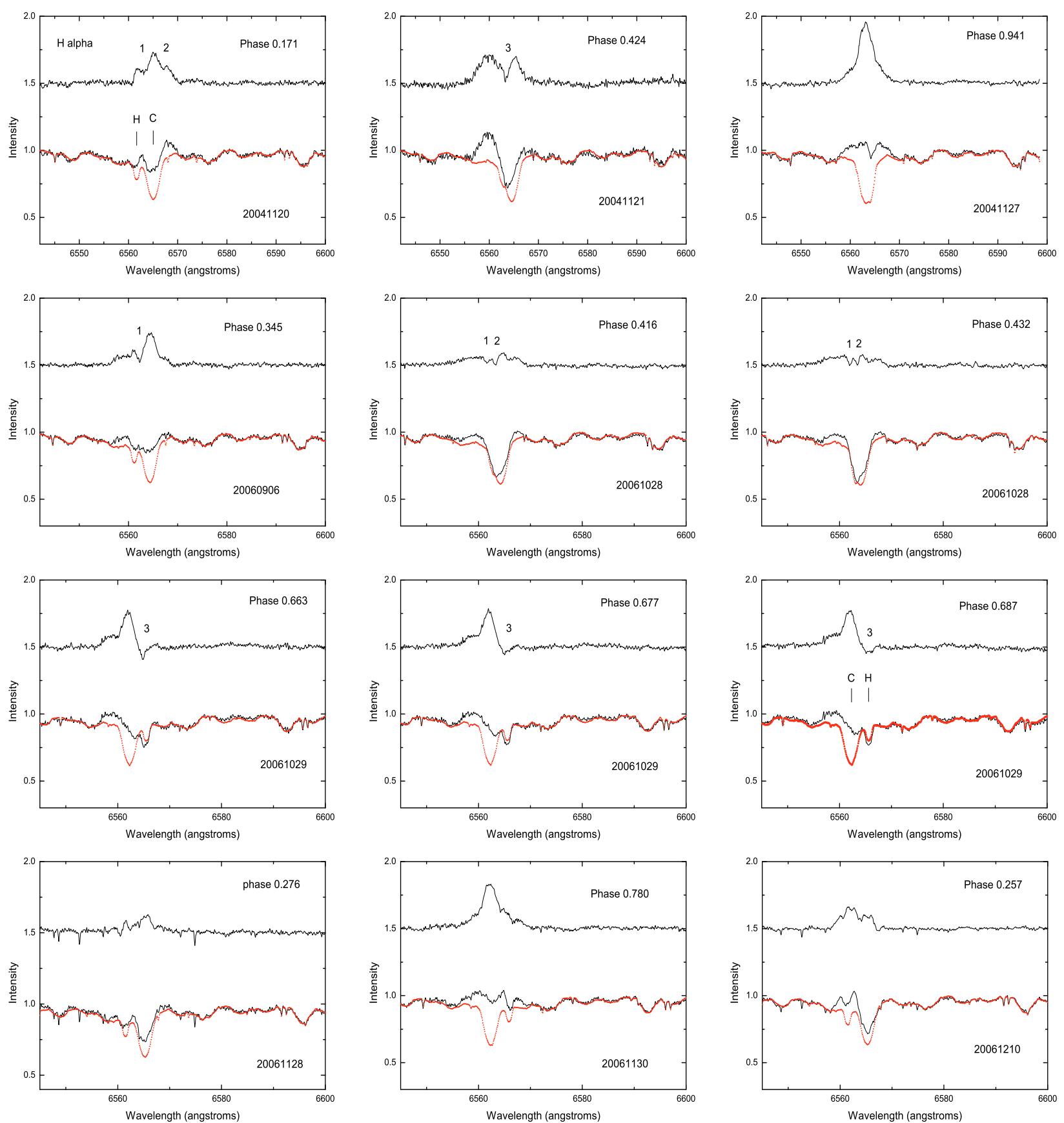

Fig. 3. Samples of the profiles of the $\mathrm{H}_{\alpha}$ lines. The dotted lines represent the synthesized spectra. The upper spectra are the subtracted spectra. The number indicates that the absorption events appeared in the excess emission profiles.

The equivalent widths $(E W \mathrm{~s})$ of the excess emissions were measured using the IRAF SPLOT task. When the profiles of two components are easily separated, the $E W \mathrm{~s}$ were evaluated on the subtracted spectra by integrating over the emission profile. In addition, we measured the $E W$ s using the Gaussian fit (For asymmetric profiles, we used several Gaussian profiles to fit), If the profiles are blended, we measured the $E W \mathrm{~s}$ using several Gaussian fits. For comparison, we also measured the $E W \mathrm{~s}$ with the Lorenzian fit. The errors of the $E W \mathrm{~s}$ were estimated by using the difference between the results of these different methods. The net $E W \mathrm{~s}$ of the excess emissions are listed in Table 2.

Because of nearly a 4 integral-day period for SZ Psc, it was very difficult to observe effectively, and the phase coverage interval of observation was short per night at a single site, so we only had a few spectra per night. To discuss the rotational modulation of chromospheric activity, we used all of the 2006 data because we only have 3 to 4 data points per rotation in different epochs.

\subsection{The $\mathrm{Na}$ I $D_{1}, D_{2}$, and $\mathrm{He}$ I $D_{3}$ lines}

The Na I $D_{1}, D_{2}$ lines are formed in the upper photosphere and lower chromosphere. The filled-in absorption of the core of the $\mathrm{Na}$ I $\mathrm{D}_{1}, \mathrm{D}_{2}$ lines could be used as chromospheric activity indicators (Andretta et al. 1997; Monte et al. 1997).

The Na I D lines are characterized by deep absorption (see Fig. 1). The core of the lines does not show any reversal. The application of the spectral subtraction technique reveals that the 
Table 2. The measurements for the excess emissions of the Na I $\mathrm{D}_{1}, \mathrm{D}_{2}, \mathrm{H}_{\alpha}$, and Ca II IRT lines.

\begin{tabular}{cccccccc}
\hline \hline Phase & $E W_{\mathrm{Na}_{\text {I }} \mathrm{D}_{1}}(\AA)$ & $E W_{\mathrm{Na}_{\mathrm{I}} \mathrm{D}_{2}}(\AA)$ & $E W_{\mathrm{H}_{\alpha}}(\AA)$ & $E W_{\text {CaII } 8498}(\AA)$ & $E W_{\text {CaII } 8542}(\AA)$ & $E W_{\text {CaII }_{\text {II }} 8662}(\AA)$ & $E W_{8542} / E W_{8498}$ \\
\hline 0.171 & $0.067 \pm 0.006$ & - & $1.053 \pm 0.114$ & $0.798 \pm 0.002$ & $1.224 \pm 0.025$ & $0.932 \pm 0.016$ & $1.534 \pm 0.027$ \\
0.424 & $0.149 \pm 0.020$ & $0.066 \pm 0.014$ & $1.503 \pm 0.020$ & $0.796 \pm 0.025$ & $1.361 \pm 0.036$ & $0.986 \pm 0.007$ & $1.710 \pm 0.008$ \\
0.941 & $0.080 \pm 0.008$ & $0.049 \pm 0.003$ & $1.908 \pm 0.004$ & $0.827 \pm 0.002$ & $1.425 \pm 0.039$ & $1.030 \pm 0.017$ & $1.723 \pm 0.043$ \\
0.092 & $0.185 \pm 0.001$ & $0.090 \pm 0.005$ & $1.284 \pm 0.005$ & $0.706 \pm 0.027$ & $1.328 \pm 0.083$ & $0.949 \pm 0.012$ & $1.881 \pm 0.046$ \\
0.857 & $0.212 \pm 0.010$ & $0.121 \pm 0.015$ & $1.607 \pm 0.039$ & $0.752 \pm 0.032$ & $1.294 \pm 0.045$ & $0.969 \pm 0.012$ & $1.721 \pm 0.013$ \\
0.094 & $0.203 \pm 0.004$ & $0.104 \pm 0.013$ & $1.309 \pm 0.019$ & $0.694 \pm 0.026$ & $1.318 \pm 0.067$ & $0.948 \pm 0.002$ & $1.899 \pm 0.025$ \\
0.345 & $0.150 \pm 0.023$ & $0.101 \pm 0.003$ & $1.004 \pm 0.011$ & $0.697 \pm 0.013$ & $1.254 \pm 0.050$ & $0.921 \pm 0.011$ & $1.799 \pm 0.038$ \\
0.416 & $0.234 \pm 0.017$ & $0.052 \pm 0.004$ & $0.653 \pm 0.010$ & $0.718 \pm 0.019$ & $1.160 \pm 0.038$ & $0.789 \pm 0.010$ & $1.616 \pm 0.010$ \\
0.432 & $0.194 \pm 0.001$ & $0.052 \pm 0.010$ & $0.637 \pm 0.013$ & $0.726 \pm 0.002$ & $1.204 \pm 0.019$ & $0.830 \pm 0.006$ & $1.658 \pm 0.022$ \\
0.663 & - & - & $0.880 \pm 0.030$ & $0.724 \pm 0.017$ & $1.202 \pm 0.013$ & $0.935 \pm 0.003$ & $1.660 \pm 0.021$ \\
0.677 & - & - & $0.934 \pm 0.031$ & $0.689 \pm 0.019$ & $1.192 \pm 0.007$ & $0.967 \pm 0.009$ & $1.730 \pm 0.038$ \\
0.687 & - & - & $0.836 \pm 0.010$ & $0.689 \pm 0.021$ & $1.176 \pm 0.005$ & $0.969 \pm 0.015$ & $1.707 \pm 0.045$ \\
0.241 & $0.068 \pm 0.005$ & $0.031 \pm 0.002$ & $0.583 \pm 0.002$ & $0.688 \pm 0.015$ & $1.367 \pm 0.018$ & $0.921 \pm 0.010$ & $1.987 \pm 0.017$ \\
0.276 & - & - & $0.576 \pm 0.014$ & $0.759 \pm 0.019$ & $1.330 \pm 0.012$ & $0.910 \pm 0.002$ & $1.752 \pm 0.028$ \\
0.743 & $0.105 \pm 0.004$ & $0.072 \pm 0.004$ & $1.663 \pm 0.005$ & $0.716 \pm 0.041$ & $1.391 \pm 0.037$ & $0.990 \pm 0.004$ & $1.943 \pm 0.060$ \\
0.780 & $0.087 \pm 0.002$ & $0.074 \pm 0.006$ & $1.715 \pm 0.005$ & $0.706 \pm 0.026$ & $1.349 \pm 0.038$ & $0.991 \pm 0.028$ & $1.911 \pm 0.017$ \\
0.753 & $0.123 \pm 0.008$ & $0.092 \pm 0.008$ & $1.761 \pm 0.036$ & $0.726 \pm 0.038$ & $1.431 \pm 0.021$ & $1.011 \pm 0.010$ & $1.971 \pm 0.074$ \\
0.794 & - & - & $1.760 \pm 0.013$ & $0.678 \pm 0.031$ & $1.426 \pm 0.003$ & $0.992 \pm 0.023$ & $2.103 \pm 0.092$ \\
0.257 & - & - & $0.775 \pm 0.016$ & $0.710 \pm 0.011$ & $1.368 \pm 0.030$ & $0.933 \pm 0.003$ & $1.927 \pm 0.012$ \\
0.294 & - & - & $0.850 \pm 0.035$ & $0.748 \pm 0.016$ & $1.370 \pm 0.031$ & $0.912 \pm 0.020$ & $1.832 \pm 0.002$ \\
\hline
\end{tabular}

“_" No emission is detected.

cores of the $\mathrm{Na} \mathrm{I}_{1}, \mathrm{D}_{2}$ lines are weak filled-in absorption for the cooler component. In some cases, the $\mathrm{Na} I \mathrm{D}_{1}, \mathrm{D}_{2}$ lines exhibit obvious excess emission from the cooler component.

The measured $E W \mathrm{~s}$ of the excess emissions in the $\mathrm{NaI}$ $\mathrm{D}_{1}$, line are plotted vs. the orbital phase in Fig. 4. For $\mathrm{Na}$ I $\mathrm{D}_{1}$, it seems that there are two minima around the quadratures. That suggests an active longitude phenomenon during our observations.

The He $\mathrm{I}_{3}$ line is formed in the upper chromosphere and is an activity indicator for the Sun and late-type stars. The emission of the line is a probe for detecting flare-like events (Zirin 1988). In our set of 20 spectra in two different observing seasons, we observed no flare-like episodes.

\subsection{The Ca II IRT lines}

The Ca II IRT lines are important chromospheric activity indicators for the Sun and late-type stars (Shine \& Linsky 1972; Shine \& Linsky 1974; Linsky et al. 1979; Foing et al. 1989; Dempsey et al. 1993; Gunn \& Doyle 1997; Montes et al. 1997, 2000; Andretta et al. 2005). They are formed in the lower chromosphere making them sensitive probes of the temperature minimum region (Montes et al. 1997).

For all the observed spectra (Fig. 1), we can see that the Ca II IRT absorption lines are from both components and that the cooler component shows strong filled-in absorption or sometimes even small self-reversal core emission. For the spectra during Nov. 28-30, 2006 and Dec. 8-10, 2006, we note that the Ca II 8498 line exhibits weak self-reversal emission in the line core, while the Ca II 8542 and 8662 lines show little or no such self-reversal core emission. For the spectra during Oct. 28, 29, 2006, especially the spectra on Oct. 28, 2006, all Ca II IRT lines exhibit obvious self-reversal in the line core. This is the strongest for the Ca II 8498 line, a little weaker for the Ca II 8542 line, and distinctly the weakest for the Ca II 8662 line.

The spectral subtraction reveals that the cooler component shows clear Ca II IRT excess emission. For the subtracted spectra, we measured the $E W$ s of the excess emission and plotted vs. orbital phase in Fig. 4. From this figure, we find no significant

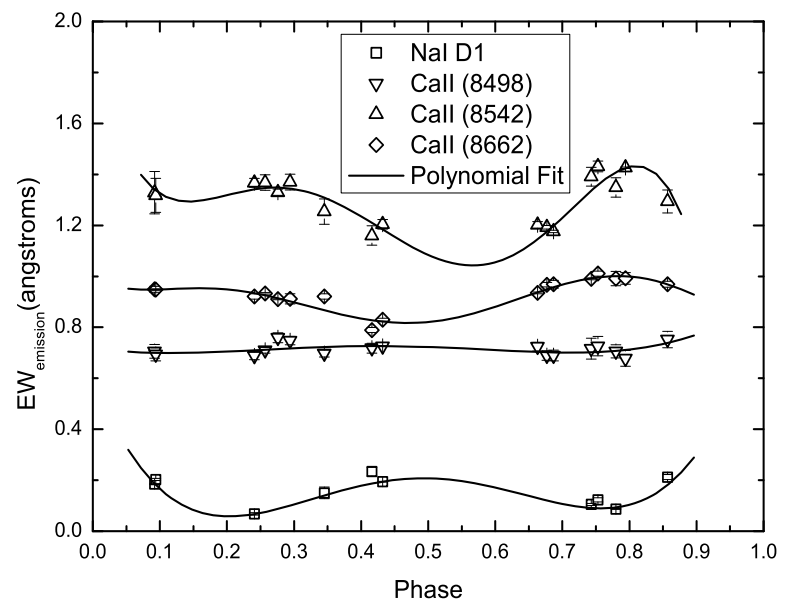

Fig. 4. The $E W$ s of the excess emissions vs. orbital phase for $\mathrm{Na}$ I D and Ca II IRT lines. The solid line refers to a polynomial fit to the data.

trend in the Ca II 8498 data. For the Ca II 8542 and 8662 lines, especially for the Ca II 8542 line, it seems that there are two active longitudes near phases 0.25 and 0.75 . In addition, the excess emission is also stronger (within the errors) at phase 0.09 .

\subsection{The $H_{\alpha}$ line}

The $\mathrm{H}_{\alpha}$ line is a very useful indicator of chromospheric activity, and it is formed in the middle chromosphere (Montes et al. 1997, etc.).

The $\mathrm{H}_{\alpha}$ line exhibits variation from absorption to weak emission (see Fig. 1). Also, it is usually filled in with broad emission. All the subtracted $\mathrm{H}_{\alpha}$ spectra indicate that the cooler component is active. Some excess emission profiles are broader and centered on the cooler component, for example, the spectra at phases 0.941 and 0.780 . Furthermore, there are obvious absorption features in the subtracted spectra, at orbital phases 0.171 , $0.424,0.345,0.663,0.677$, and 0.687 . The $E W$ s of the excess emissions for the 2006 data are plotted against orbital phase in 


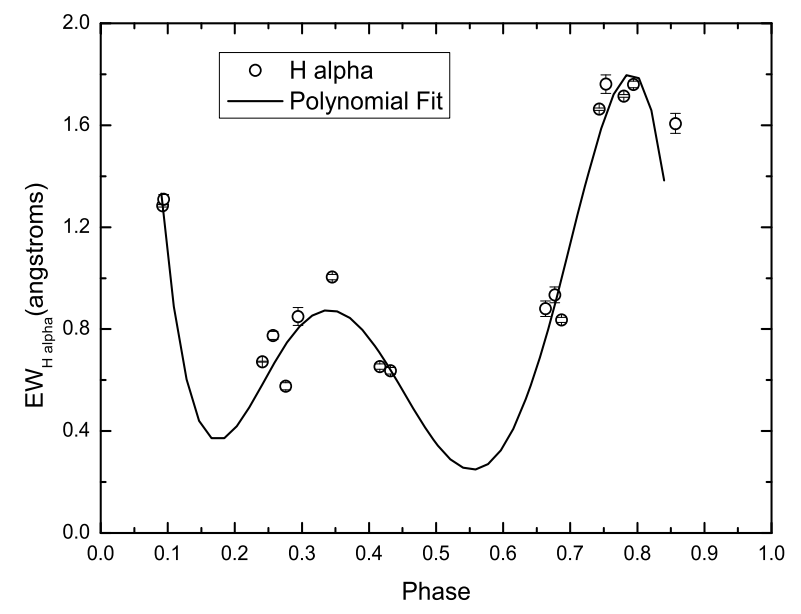

Fig. 5. As Fig. 4, but for the $E W \mathrm{~s}$ of $\mathrm{H}_{\alpha}$ line.

Fig. 5. The orbital modulation of the $\mathrm{H}_{\alpha}$ excess emission is similar to Ca II 8542 and 8662 data. It seems that there are also two active longitudes near phases 0.35 and 0.75 .

\section{Discussion}

In this paper, we analyze simultaneous spectroscopic observations of several optical chromospheric activity indicators for the RS CVn eclipsing binary system SZ Psc using the spectral subtraction technique. We investigate the details of the excess emission and study the chromospheric emission variation with orbital phase.

\subsection{Chromospheric activity}

For the Ca II IRT lines, we find the cooler component of SZ Psc shows filled-in absorption or sometimes even weak self-reversal core emission. This might be due to the low-level plage and high rotational velocity of the cooler component of SZ Psc. For some other chromospherically active binary systems, Montes et al. (2000) also detected a similar feature in the Ca II IRT lines.

For the most obvious self-reversal core emission feature, it is normal that the reversal is the strongest for the Ca II 8498 line, a little weaker for the Ca II 8542 line, and weakest of all for the Ca II 8662 line. This is consistent with the observation of the CaII IRT lines of solar plages with different degrees of activity (Shine \& Linsky 1972; Shine 1973; Shine \& Linsky 1974). The increase in self-reversal core emission in the Ca II IRT lines results from the higher temperatures and higher electron densities at a given line optical depth produced by the steeper temperature gradient (Shine \& Linsky 1974).

For the ratio between $E W_{8542}$ and $E W_{8498}$, we find the values are generally low, between $1-3$, which indicates optically thick emission in plage-like regions. In solar plage, the values of $E W_{8542} / E W_{8498}$ are in the range 1.5-3 (Chester 1991). These low values are also found in other chromospherically active binaries by other authors: Lázaro \& Arévalo (1997); Arévalo \& Lázaro (1999); Montes et al. (2000); and Gu et al. (2002).

All the $\mathrm{H}_{\alpha}$ spectra indicate that the cooler component is active. In the subtracted spectra, the excess emission profiles exhibit broad wings. The broad emission feature coincides with the results derived by other authors: Huenemoerder \& Ramsey (1984), Eaton \& Henry (2007), etc. There are a couple of possible explanations for this. First, the broad component could be interpreted as arising from microflaring. Such broad
$\mathrm{H}_{\alpha}$ components are also detected in some other chromospherically active stars (Hatzes 1995; Montes et al. 2000; etc). Second, it might be caused by instantaneous mass transfer from the cooler component to the hotter one (Bopp 1981). Because the cooler component is filling $82 \%$ of its Roche lobe (Lanza et al. 2001), it is very possible that the mass transfer can happen due to the strong chromospheric activity.

In summary, all the analyzed activity indicators show that the cooler component is active. This is consistent with the result that the $\mathrm{Mg}$ II h\&k emission lines are mainly enhanced by the cooler component of the system (Doyle et al. 1994a,b; Kang et al. 2003). Moreover, for the Ca II H and K lines of SZ Psc derived by Fernáandez-Figueroa et al. (1986) and Fernáandez-Figueroa et al. (1994), there is obvious emission from the cooler component and no emission from the hotter one.

\subsection{Chromospheric activity modulation}

For the non-eclipsed configuration, the modulation of excess emission with phase indicates the appearance and disappearance of discrete active regions as the star is presenting different hemispheres to us.

For the cooler component, our result suggests the presence of an active longitude phenomenon during the 2006 epoch. To demonstrate the possible rotational modulation well, we used polynomial function to fit the $E W$ s of $\mathrm{Na} \mathrm{I}_{1}, \mathrm{Ca}$ II IRT, and $\mathrm{H}_{\alpha}$ excess emission profiles, which are displayed in Figs. 4 and 5. For the CaII 8542 and 8662 and $\mathrm{H}_{\alpha}$ lines, the excess emissions (with the orbital phase) may be correlated basically, especially around two quadratures. It seems that the emissions are stronger around two quadratures of the system (phases 0.25 and 0.75 ). However, for the $\mathrm{Na} \mathrm{I}_{1}$ line, it seems that the emission is weaker around the two quadratures, so the $\mathrm{Na} \mathrm{I} \mathrm{D}_{1}$ line may be anti-correlated with the Ca II 8542 and 8662 and the $\mathrm{H}_{\alpha}$ lines. The reason might be that the $\mathrm{Na}$ I lines could be affected by the presence of active spot regions, because the reactions of neutral atoms in their ground states are very sensitive to the photospheric temperature structure (Barradoy Navascués et al. 2001). Moreover, there is some evidence that two variable active regions appear around longitudes $50^{\circ}$ and $270^{\circ}$ by analyzing a sequence of $V$-band light curves of SZ Psc (Lanza et al. 2001). In addition, both active longitudes were also suggested by Huenemoerder \& Ramsey (1984).

For another chromospherically active binary UX Ari, the favorite active longitudes are also around two quadratures of the system based on the analysis of several chromospheric activity indicators $\mathrm{He} \mathrm{I} \mathrm{D}_{3}, \mathrm{Na} \mathrm{I} \mathrm{D}_{1}, \mathrm{D}_{2}, \mathrm{H}_{\alpha}$, and CaII IRT lines $(\mathrm{Gu}$ 2005). These active longitude features have also been found using photometry on many other active RS CVn binary systems, such as EI Eri, $\sigma$ Gem, HK Lac, and RT And (Olah Hall \& Henry 1991; Henry et al. 1996; Berdyugina \& Tuominen 1998; and Zhang \& Gu 2007).

\subsection{The absorption features in $H_{\alpha}$ line.}

The interesting result from our high-resolution $\mathrm{H}_{\alpha}$ subtracted spectra is the detection of absorption components at some orbital phases in our observations. The physical properties of the absorptions are listed in Table 3, which includes orbital phase, the radial velocity measured with respect to the cooler component's frame of rest, and the $E W$ of the absorption. Similar peculiarities can be found in the differential spectra of Huenemoerder \& Ramsey (1984). 
Table 3. Observed properties of the absorption features identified by Gaussian fitting.

\begin{tabular}{lllcccc}
\hline \hline Phase & absorption & $R V\left(\mathrm{~km} \mathrm{~s}^{-1}\right)$ & $E W(\AA)$ & absorption & $R V\left(\mathrm{~km} \mathrm{~s}^{-1}\right)$ & $E W(\AA)$ \\
\hline 0.171 & absorption1 & -111 & $0.124 \pm 0.012$ & absorption2 & 97 & $0.061 \pm 0.003$ \\
0.424 & absorption3 & -57 & $0.322 \pm 0.003$ & & & \\
0.345 & absorption1 & 94 & $0.322 \pm 0.003$ & & & \\
0.416 & absorption1 & -123 & $0.052 \pm 0.007$ & absorption2 & -49 & $0.069 \pm 0.001$ \\
0.432 & absorption1 & -93 & $0.064 \pm 0.005$ & absorption2 & -29 & $0.056 \pm 0.006$ \\
0.663 & absorption3 & 118 & $0.080 \pm 0.008$ & & & \\
0.677 & absorption3 & 133 & $0.067 \pm 0.005$ & & & \\
0.687 & absorption3 & 153 & $0.064 \pm 0.006$ & & & \\
\hline
\end{tabular}

" $R V$ " is the radial velocity measured with respect to the cooler component's frame of rest. Negative velocities represent blueshifts.

The absorption features may be explained by the stellar prominence. Stellar prominence results in relative absorption while passing between the stellar disk and the observer, and then scatters photons arising in the chromosphere out of the line of sight. This phenomenon was first reported by Collier Cameron \& Robinson (1989) for the rapidly rotating star AB Dor. Since then, stellar prominence has been observed as transient absorption (usually) or emission crossing the profiles of the Balmer lines, not only in single stars but also in binary systems (Hall \& Ramsey 1994; Cameron et al. 2002).

If the numbered features in Fig. 3 arise from prominences (and are not simply due to less active stellar surface areas or the velocity separation of the components.), we calculate the distances of the prominences from the rotation axis of cooler component, using the simple model (Collier Cameron \& Robinson 1989). During Oct. 28, 29, 2006, the distances of the prominences1, 2, and 3 (see Fig. 3) are $3.8 R, 2.5 R$, and $2.9 R \pm 0.5 R$ ( $R$ is the radius of the cooler component), respectively. For the prominence 3 , if we let it go back to the phase 0.432 , the velocity of prominence 3 should be $-144 \pm 51 \mathrm{~km} \mathrm{~s}^{-1}$. On the other hand, the velocity of the prominence 1 detected at phase 0.432 is just inside the range. Thus, prominencel and prominence 3 might came from the same place. During the 2004 observing season, the velocities of the two absorptions are $-111 \mathrm{~km} \mathrm{~s}^{-1}$ blueward and $97 \mathrm{~km} \mathrm{~s}^{-1}$ redward of the central velocity of the cooler component at phase 0.171 , and the velocity of the absorption 3 feature is about $57 \mathrm{~km} \mathrm{~s}^{-1}$ blueward of the cooler component at phase 0.424 . If absorption 1 and 3 have the same prominence, the distance of the prominence is $1.7 R$. The $E W$ of the absorption 3 is larger than one of the absorption 1 . This indicates that the prominence absorption should be much more visible against the disk of the cooler component from the phase 0.171 to 0.424 , due to the much greater projected area. For absorption2 at phase 0.171 , if the distance of the promience 2 is $2.7 R$ (the average value of the distances of our observed prominences), when moving to 0.424 , the radial velocity should be about $185 \mathrm{~km} \mathrm{~s}^{-1}$ redward of the cooler component. It is just the critical point of the excess emission, and absorption 2 should not be visible against the stellar disk at phase 0.424 . This is consistent with our result that there is no absorption feature at the critical point of the redward excess emission at phase 0.424 .

In the course of the spectral analysis, it was found that there are some absorption components around the primary profiles at some phases such as $0.663,0.667$, and 0.687 . These absorptions may also be a mass transfer effect since the cooler component is very close to its Roche lobe and the mass transfer occurs more easily when considering the strong magnetic activity. These phenomena are also found in other RS CVn stars by other authors: Huenemoerder et al. (1989), Hall \& Ramsey (1992), Gu et al. (2002).
Unfortunately, our data do not cover the $\mathrm{H}_{\beta}$ line. We cannot tell whether this is optically thick or thin structure via the ratio $E W_{\mathrm{H}_{\alpha}} / E W_{\mathrm{H}_{\beta}}$ (Huenemoerder et al. 1989). It is impossible to define the nature of the absorption precisely at present.

\section{Conclusions}

The results from the analysis of our spectral data of SZ Psc can be summarized as follows.

1. The chromospheric activity indicators $\mathrm{Na}$ I $\mathrm{D}_{1}, \mathrm{D}_{2}, \mathrm{Ca}$ II IRT and $\mathrm{H}_{\alpha}$ lines show that the cooler component is active in the system.

2. We find that the $E W_{8542} / E W_{8498}$ is in the range $1-3$, which indicates optically thick emission in plage-like regions.

3. For the Ca II 8542 and 8662 and the $\mathrm{H}_{\alpha}$ lines in 2006, it seems that the excess emissions are stronger near the two quadratures of the system. They may be anti-correlated with the behavior of the Na I $D_{1}$ line. These suggest the presence of the active longitude phenomenon during our observations.

4. The absorption features are detected in the subtracted $\mathrm{H}_{\alpha}$ lines, which could be explained by prominence-like extended material seen on the stellar disk or mass transfer from the cooler component to the hotter one.

Acknowledgements. The authors would like to thank the observing assistants of the $2.16 \mathrm{~m}$ telescope of Xinglong station for their help and support during our observations. We are very grateful to Dr. Montes for providing a copy of the STARMOD program. We would also like to thank the anonymous referee for his or her valuable suggestions and comments, which have led to significant improvement in our manuscript. We would also thank Prof. Chris Sterken and the team at the SWYA conference in Blankenberge, Belgium, which led to many improvements, including in the language of the revised manuscript. This work is supported by the NSFC under grants No. 10373023 and 10773027.

\section{References}

Andretta, V., Doyle, J. G., \& Byrne, P. B. 1997, A\&A 322, 266

Andretta, V., Busà, I., Gomez, M. T., \& Terranegra, L. 2005, A\&A, 430, 669

Antonopoulou, E., Deliyannis, J., \& Mitrou, C. K. 1995, Inf. Bull. Var. Stars, No. 4262

Arévalo, M. J., \& Lázaro, C. 1999, AJ, 118, 1015

Barrado y Navascués, D., García Lopez, R. J., Severino, G., \& Gomez, M. T. 2001, A\&A, 371, 652

Barden, S. C. 1985, ApJ, 295, 162

Berdyugina, S. V., \& Tuominen, I. 1998, A\&A, 336, L25

Bopp, B. W. 1981, AJ, 86, 771

Bopp, B. W., \& Talcott, J. C. 1978, AJ, 83, 1517

Catalano, S., Frisina, A., Rodono, M., \& Scaltriti, F. 1978, Inf. Bull. Var. Stars, 1427

Chester, M. M. 1991, Ph.D. Thesis, Pennsylvania State Univ.

Collier Cameron, A., \& Robinson, R. D. 1989, MNRAS, 236, 57

Collier Cameron, A., Jardine, M., \& Donati, J.-F. 2002, in Stellar Coronae in the Chandra and XMM-Newton Era, ed. F. Favata, \& J. J. Drake, ASP Conf. Ser., 277,397 
Dempsey, R. C., Bopp, B. W., Henry, G. W., \& Hall, D. S. 1993, ApJS, 86, 293 Doyle, J. G., Mitrou, C. K., Mathioudakis, M., et al. 1994a, A\&A, 291, 135 Doyle, J. G., Mitrou, C. K., Mathioudakis, M., \& Antonopoulou, E. 1994b, A\&A, 283, 522

Eaton, J. A. 1977, Inf. Bull. Var. Stars, No. 1297

Eaton, J. A., \& Hall, D. S. 1979, ApJ, 227, 907

Eaton, J. A., \& Henry, G. W. 2007, PASP, 119, 259

Eaton, J. A., Scaltriti, F., Cerruti-sola, M., et al. 1982, Ap\&SS, 82, 289

Fernández-Figueroa, M. J., De Castro, E., Montesinos, B., et al. 1986, Adv. Space. Res., 6, 187

Fernández-Figueroa, M. J., Montes, D., De Castro, E., \& Cornide, M. 1994 ApJS, 90, 433

Foing, B., Crivellari, L., Vladilo, G., Rebolo, R., \& Beckman, J. 1989, A\&AS, 80,189

Frasca, A., \& Catalano, S. 1994, A\&A, 284, 883

Frasca, A., Marino, G., Catalano, S., \& Marilli, E. 2000, A\&A, 358, 1007

Gálvez, M. C., Montes, D., Fernández-Figueroa, M. J., De Castro, E., \& Cornide, M. 2007, A\&A, 472, 587

Gray, R. O., Napier, M. G., \& Winkler, L. I. 2001, AJ, 121, 2148

Gu, S.-H. 2005, in Coronal and Stellar Mass Ejections, IAU Symp. Proc. 226, Held 13-17 September, Beijing, ed. K. Dere, J. Wang, \& Y. Yan (Cambridge: Cambridge University Press), 501

Gu, S.-H., Tan, H.-S., Shan, H.-G., \& Zhang, F.-H. 2002, A\&A, 388, 889

Gunn, A. G., \& Doyle, J. G. 1997, A\&A, 318, 60

Gunn, A. G., Doyle, J. G., \& Houdebine, E. R. 1997, A\&A, 319, 211

Hall, D. S. 1976, in Multiple Periodic Variable Stars, ed. W. Fitch (Dordrecht: Reidel), 287

Hall, J. C., \& Ramsey, L. W. 1992, AJ, 104, 1942

Hall, J. C., \& Ramsey, L. W. 1994, AJ, 107, 1149
Hatzes, A. P. 1995, AJ, 109, 350

Henry, G. W., Eaton, J. A., Hamer, J., et al. 1996, ApJ, 462, 888

Huenemoerder, D. P., \& Ramsey, L. W. 1984, AJ, 89, 549

Huenemoerder, D. P., Buzasi, D. L., \& Ramsey, L. W. 1989, AJ, 98, 1398

Jakate, S. M., Bakos, G. A., Fernie, J. D., \& Heard, J. F. 1976, AJ, 81, 250

Jensch, A. 1934, Astron. Nachr., 252, 393

Kang, Y. W., Lee, W.-B., Kim, H.-I., \& Oh, K.-D. 2003, MNRAS, 344, 1227

Lanza, A., Rodonò, M., Mazzola, L., \& Messina, S. 2001, A\&A, 376, 1011

Lázaro, C., \& Arévalo, M. J. 1997, AJ, 113, 2283

Linsky, J. L., Hunten, D., Glacken, D., \& Kelch, W. 1979, ApJS, 41, 481

Montes, D., Fernández-Figueroa, M. J., De Castro, E., \& Cornide, M. 1995, A\&A, 294, 165

Montes, D., Fernández-Figueroa, M. J., De Castro, E., \& Sanz-Forcada, J. 1997, A\&AS, 125,263

Montes, D., Fernández-Figueroa, M. J., De Castro, E., et al. 2000, A\&AS, 146, 103

Popper, D. M. 1988, AJ, 96, 1040

Ramsey, L. W., \& Nations, H. L. 1981, PASP, 93, 732

Shan, H. G., Liu, X. F., \& Gu, S. H. 2006, NA, 11, 287

Shine, R. A. 1973, Ph.D. Thesis, University of Colorado

Shine, R. A., \& Linsky, J. L. 1972, SP, 25, 357

Shine, R. A., \& Linsky, J. L. 1974, SP, 39, 49

Strassmeier, K. G., Hall, D. S., Fekel, F. C., \& Scheck, M. 1993, A\&AS, 100, 173

Tunca, Z. 1984, Ap\&SS, 105, 23

Weiler, E. J. 1978, MNRAS, 182, 77

Wright, J. T., Marcy, G. W., Butler, R. P., \& Voght, S. S. 2004, ApJS, 152, 261

Zirin, H. 1988, in Astrophysics of the Sun (Cambridge University Press)

Zhang, L. Y., \& Gu, S. H. 2007, A\&A, 471, 219 